\title{
Article \\ Factorization à la Dirac Applied to Some Equations of Classical Physics
}

\author{
Zine El Abiddine Fellah $^{1, *}$, Erick Ogam ${ }^{1}{ }^{\mathbb{D}}$, Mohamed Fellah ${ }^{2}$ and Claude Depollier ${ }^{3,4}$ \\ 1 LMA, CNRS, UPR 7051, Centrale Marseille, Aix-Marseille University, CEDEX 20, F-13402 Marseille, France; \\ ogam@lma.cnrs-mrs.fr \\ 2 Laboratoire de Physique Théorique, Faculté de Physique, USTHB, BP 32 El Alia, Bab Ezzouar 16111, Algeria; \\ fellahm1@yahoo.fr \\ 3 UMR CNRS 6613, Laboratoire d'Acoustique de l'Universite du Maine, LUNAM Universite du Maine, \\ UFR STS Avenue O. Messiaen, CEDEX 09, 72085 Le Mans, France; Claude.Depollier@univ-lemans.fr \\ 4 ESST, 43 Chemin Sidi M'Barek, Oued Romane 16104, El Achour, Algeria \\ * Correspondence: Fellah@lma.cnrs-mrs.fr
}

check for

updates

Citation: Fellah, Z.E.A.; Ogam, E.; Fellah, M.; Depollier, C. Factorization à la Dirac Applied to Some Equations of Classical Physics. Mathematics 2021, 9, 899. https://doi.org/10.3390/ math9080899

Academic Editor: Renat T. Sibatov

Received: 14 February 2021

Accepted: 13 April 2021

Published: 18 April 2021

Publisher's Note: MDPI stays neutral with regard to jurisdictional claims in published maps and institutional affiliations.

Copyright: (c) 2021 by the authors. Licensee MDPI, Basel, Switzerland. This article is an open access article distributed under the terms and conditions of the Creative Commons Attribution (CC BY) license (https:// creativecommons.org/licenses/by/ $4.0 /)$.

\begin{abstract}
In this paper, we present an application of Dirac's factorization method to three types of the partial differential equations, i.e., the wave equation, the scattering equation, and the telegrapher's equation. This method gives results that contribute to a better understanding of physical phenomena by generalizing the Euler and constituent equations. Its application to the wave equation shows that it is indeed a factorization method, since it gives d'Alembert's solutions in a more general framework. In the case of the diffusion equation, a fractional differential equation has been established that has already been highlighted by other authors in particular cases, but by indirect methods. Dirac's method brings several new results in the case of the telegraphers' equation corresponding to the propagation of an acoustic wave in a dissipative fluid. On the one hand, its formalism facilitates the temporal interpretation of phenomena, in particular the density and compressibility of the fluid become temporal operators, which can be "seen" as susceptibilities of the fluid. On the other hand, a consequence of this temporal modeling is the highlighting in Euler's equation of a term similar to the one that was introduced by Boussinesq and Basset in the equation of the motion of a solid sphere in a unsteady fluid.
\end{abstract}

Keywords: factorization method; diffusion equation; wave equation; telegraph equation; fractional calculus; basset force

\section{Introduction}

The factorization method has been developed to solve the ordinary differential equations that are encountered in many fields of physics and more particularly in quantum mechanics and in classical fields, such as acoustics and electromagnetism. Initially, it is a procedure that provides an answer to eigenvalue problems. The factorization method applied to the differential problem

$$
L(D) u=0,
$$

where $L$ is a differential operator with constant coefficients, $D$ is the set of relevant partial derivatives in the equation and $u$ a distribution, consists in finding the $P$ and $Q$ operators, such as when $L$ is factorized as

$$
L(D)=P(D) Q(D)
$$

it is possible to find a general solution to (1) in the form of

$$
u=u_{1}+u_{2}
$$


$u_{1}$ and $u_{2}$ being solutions that correspond to each equation:

$$
P(D) u_{1}=0, \quad Q(D) u_{2}=0 \quad \text { in } \quad \Omega,
$$

where $\Omega$ is an open set in $\mathbb{R}^{n}$. It is clear that $u_{1}$ and $u_{2}$ verifying (4) are solutions to (1). However, any solution to (1) can only be put in the form (3), provided that the factorization (2) and domain $\Omega$ are specified [1].

Schrödinger was one of the initiators of the factorization method, who applied what he called the "first-order adjoint operator method" to the case of the hypergeometric equation [2,3]. It is inspired by Dirac's creation-annihilation operators approach [4] and by Pauli and Weyl's ideas on spherical harmonics with spin.

Subsequently, Infeld and Hull proposed a different factorization method [5-11]. The literature on this subject has grown considerably since then. For example, to cite just a few authors, reference can be made to Mielnik [12,13], Gendenshtein [14], Smirnov [15,16], and Rosu $[17,18]$. Let us also mention that part of this work is at the origin of the ideas behind the theory of supersymmetry.

Let us recall that Dirac's theory stems from his preoccupation with establishing, from the Klein-Gordon equation, the analogue for the theory of the electron of Maxwell's equations of electromagnetism. In other words, Dirac's goal was to show the relations between the components of the $1 / 2$ spin field [19].

The method that was developed by Dattoli and his collaborators [20-24] subsequently noted Dirac's method or DM, replacing a second-order differential equation with a pair of first-order differential equations. It generalizes the classical factorization method on several levels. First of all, it applies very naturally to second order partial differential equations, which makes it convenient and efficient for the search for solutions of wave equations, telegrapher's equations and generalized diffusion equations. Another advantage of the method is that it leads to several forms of solutions. By its nature, it results in a system of lower order equations that are either independent from or coupled to one another. In addition, it can be generalized to equations of order higher than 2 and, for starting equations having odd order derivatives, it leads to differential equations that contain fractional derivatives [25]. Therefore, the latter takes into account the memory effects that develop during the evolution of the processes studied. This is an interesting aspect when we know that memory effects generally have a strong influence on transport, leading, for example, to non-stationarity. All of these advantages make Dirac factorization an effective method for the interpretation of physical processes.

Our goal here is not to solve differential equations, but to show that Dirac's method makes it possible to either highlight hidden properties, such as those of conservation laws, in the form of new equations that are linked to the previous ones, or to find by a simpler method and already known equation. In this paper, we propose an application of this method to the partial differential equations that are encountered in fluid mechanics, namely the wave equation, the viscous diffusion equation, and the telegraph's equation

The outline of the paper is the following. Section 2, for the sake of clarity, gives a brief summary on Dirac's method, recalls some definitions and properties of fractional derivatives, and provides the derivation of the investigated equations. In Section 3, applications of DM show that it leads to results, some already known that have been obtained by other methods, and some newer ones that lead to original physical interpretations of the processes. Section 4 is devoted to our conclusion.

\section{Basic Tools}

In this section, in the interest of completeness, we review fundamental concepts and some results of prior studies that are relevant for our discussion. 


\subsection{Dirac Like Method}

The DM can be summarized, as follows. Let the sum of two squared operators $A$ and $B$ be:

$$
A^{2}+B^{2}
$$

The DM suggests that the operator $\mathcal{O}$, defined as the square root of $A^{2}+B^{2}$ :

$$
\mathcal{O}=\sqrt{A^{2}+B^{2}}
$$

can be written as follows:

$$
\mathcal{O}=\alpha A+\beta B
$$

Therefore it is obvious that the mathematical elements $\alpha$ and $\beta$ are not "ordinary numbers". For this equality to be meaningful, it is necessary that

$$
(\alpha A+\beta B)^{2}=A^{2}+B^{2},
$$

which implies that the following conditions must be satisfied :

$$
\begin{aligned}
& \alpha^{2}=\beta^{2}=1 \\
& \alpha \beta+\beta \alpha=0 .
\end{aligned}
$$

Pauli's matrices:

$$
\sigma_{1}=\left(\begin{array}{ll}
0 & 1 \\
1 & 0
\end{array}\right), \sigma_{2}=\left(\begin{array}{cc}
0 & -i \\
i & 0
\end{array}\right), \sigma_{3}=\left(\begin{array}{cc}
1 & 0 \\
0 & -1
\end{array}\right)
$$

checking the following relationships

$$
\begin{aligned}
\sigma_{i} \sigma_{j} & =i \varepsilon_{i j k} \sigma_{k}+\delta_{i j} I_{2}, \\
\left\{\sigma_{i}, \sigma_{j}\right\} & =2 \delta_{i j} I_{2},
\end{aligned}
$$

where $I_{2}$ is the unit matrix $2 \times 2$ and $\varepsilon_{j k l}$ is the Levi-Civita symbol defined as totally antisymmetric in all three indices, comply with the conditions (9) and (10).

In this framework, the operator $\mathcal{O}$ that is defined by (7) has the structure of a Pauli vector and is given by the expression :

$$
\mathcal{O}=A \sigma_{k}+B \sigma_{l}, \quad \text { with } \quad k, l \in\{1,2,3\} \quad \text { and } \quad k \neq l
$$

A choice of different couples $(k, l)$ leads to different solutions to the problem under interest. Below, we will see that this property is one of DM's assets.

\subsection{Fractional Calculus}

The definition of the fractional derivative follows naturally from that of the fractional integral. The fractional integral noted ${ }_{0} I_{t}^{\alpha} f(t)$ is the straightforward generalization of the standard n-fold primitive of $f(t)$ :

${ }_{0} I_{t}^{n} f(t)=\int_{0}^{t} \int_{0}^{t_{n-1}} \ldots \int_{0}^{t_{1}} f\left(t_{0}\right) d t_{0} \ldots d t_{n-1}=\frac{1}{(n-1) !} \int_{0}^{t}(t-\tau)^{n-1} f(\tau) d \tau \quad n \in \mathbb{N}$.

Thus, we have

$$
{ }_{0} I_{\alpha}^{\alpha} f(t)=\frac{1}{\Gamma(\alpha)} \int_{0}^{t}(t-\tau)^{\alpha-1} f(\tau) d \tau, \quad \alpha \in \mathbb{R}^{+}
$$

where $\Gamma(\dot{)}$ is the Gamma function. For the sake of convenience, for the following we introduce the function

$$
\Phi_{\alpha}(t)=\frac{t_{+}^{\alpha-1}}{\Gamma(\alpha)}, \quad \alpha>0,
$$


where the subscript + means that $t^{\alpha-1}$ is a causal function with the convolution composition rule

$$
\Phi_{\alpha}(t) * \Phi_{\beta}(t)=\Phi_{\alpha+\beta}(t) .
$$

Thus, the fractional integral of order $\alpha>0$ can be considered to be the convolution between and $f(t)$ and $\Phi_{\alpha}(t)$ :

$$
{ }_{0} I_{t}^{\alpha} f(t)=\Phi_{\alpha}(t) * f(t)
$$

Because derivation and integration operations are the inverse of each other, the functional relationship

$$
D_{t}^{m} \circ{ }_{0} I_{t}^{m} f(t)=f(t) \quad t>0,
$$

where $D_{t}^{m}$ is the derivative operator $D_{t}^{m}=d^{m} / d t^{m}$, makes it possible to determine the Riemann-Liouville fractional derivative of order $\alpha>0$ as ${ }_{0}^{R L} D_{t}^{\alpha}=D_{t}^{m} \circ{ }_{0} I_{t}^{m-\alpha} f(t)$ i.e.,

$$
{ }_{0}^{R L} D_{t}^{\alpha} f(t)=\frac{1}{\Gamma(m-\alpha)} \frac{d^{m}}{d t^{m}} \int_{0}^{t} \frac{f(\tau)}{(t-\tau)^{\alpha+1-m}} d \tau, \quad m-1<\alpha<m .
$$

This derivative has the following standard properties of an integer derivative:

$$
\begin{aligned}
{ }_{0}^{R L} D_{t}^{\alpha} \circ{ }_{0} I_{t}^{\alpha}= & D_{t}^{m} \circ_{0} I_{t}^{m-\alpha} \circ_{0} I_{t}^{\alpha}=D_{t}^{m} \circ_{0} I_{t}^{m}=I, \\
& { }_{0}^{R L} D_{t}^{\alpha} t^{\gamma}=\frac{\Gamma(\gamma+1)}{\Gamma(\gamma+1-\alpha)} t^{\gamma-\alpha} .
\end{aligned}
$$

However, because of the poles of the function $\Gamma(z)$ for $z=0,-1,-2, \ldots$, in contrast to the integer derivative, the fractional derivative of a constant $C$ is not zero:

$$
{ }_{0}^{R L} D_{t}^{\alpha} C=C \frac{t^{-\alpha}}{\Gamma(1-\alpha)} \quad \alpha \geq 0 .
$$

To overcome this drawback, Caputo introduced the so-called Caputo fractional derivative that is defined by:

$$
{ }_{0}^{C} D_{t}^{\alpha} f(t)={ }_{0} I_{t}^{m-\alpha} \circ D_{t}^{m} f(t), \quad m-1<\alpha \leq m,
$$

i.e.,

$$
{ }_{0}^{C} D_{t}^{\alpha} f(t)=\frac{1}{\Gamma(m-\alpha)} \int_{0}^{t} \frac{f^{(m)}(\tau)}{(t-\tau)^{\alpha+1-m}} d \tau=\Phi_{m-\alpha}(t) * f^{(m)} .
$$

The fractional derivative of Caputo has two practical properties for applications: (i) ${ }_{0}^{C} D_{t}^{\alpha} C=0$ and (ii) Laplace's transform of the Caputo derivative of a function only involves integer derivatives of that function.

Thus, the fractional derivative being defined by an integral takes the history of the function to which it is applied into account. Therefore, it is an adequate tool to study the memory effects that develop in physical processes.

\subsection{Governing Equations}

In this section, the principles underlying the establishment of the wave equation, the diffusion equation, and the telegrapher's equation are recalled. In particular, it is stressed that there are two essentially different versions of the telegrapher's equation, depending on whether it is induced from the diffusion equation or from the wave equation.

\subsubsection{Wave Equation}

In various domains of physics, there are several ways to establish the equations that govern the behavior of fields. The Lagrangian method is the most general one. It is based on the general principles of physics, i.e., symmetries or invariances by translation in space and time, by rotation and other transformations in accordance with the principles of physics and the Euler-Lagrange equations. This property has been widely used to 
test the consistency of phenomenological equations [26] and in the theory of interacting particles and fields. This is the case, for example, of gauge fields [27] or Biot's theory of saturated porous media [28]. However, the Lagrangian method does not make it possible to introduce the attenuation terms in the resulting Euler equations.

In classical physics, it is customary to use a shortcut to derive the wave equations. To do this, we start from a system of two equations: (i) a balance equation of a physical quantity $n$, such as the mass, the electric charge, the heat, etc., and (ii) a constitutive relation. The latter expresses the evolution of the quantity in question under the action of the driving force that generates the process. Unlike the first equation, which is imposed by the principles of physics, the second equation depends on the mathematical model that is chosen to describe the process involved.

For instance, in linear fluid acoustics, the system of equations consists of the mass conservation equation, which takes the compressibility $K$ of the fluid and the Euler equation into account [29].

$$
\left\{\begin{array}{l}
K \partial p / \partial t=-\partial v_{i} / \partial x_{i} \\
\rho \partial v_{i} / \partial t=-\partial p / \partial x_{i}
\end{array}\right.
$$

where $p(x, t)$ and $v(x, t)$ are the pressure and velocity of the fluid, respectively. The result is a PDE of order 2 , which is the wave equation

$$
\partial^{2} f(x, t) / \partial t^{2}-c^{2} \partial^{2} f(x, t) / \partial x^{2}=0,
$$

where $f(x, t)$ is $v(x, t)$ or $p(x, t)$ and $c^{2}=1 / K \rho$.

\subsubsection{Diffusion Equation}

The diffusion equation differs from the wave equation, in that the constitutive relationship is a Fick's law involving the quantity $n$ in question. Fick's law is a linear relationship between the flux $\mathbf{q}$ of this quantity and the driving force proportional to its gradient $\nabla n$. It is a generalization of Fourier's law of heat. Subsequently, derive the diffusion equation from the following system:

$$
\left\{\begin{array}{c}
\partial n / \partial t=-\boldsymbol{\nabla} \cdot \mathbf{q} \\
\mathbf{q}=-k \boldsymbol{\nabla} n
\end{array}\right.
$$

When the diffusion develops along the $x$ axis, then $n$ is a function that only depends on $x$ and time $t, n(x, t)$. The combination of the previous equations leads to

$$
\partial n / \partial t=k \partial^{2} n / \partial x^{2} .
$$

The result is that the diffusion equation is a PDE of order 1 in time and order 2 in space. Let us note that the wave equation requires the assumption of two quantities, (e.g., pressure and velocity) in acoustics, whereas the diffusion equation only assumes the function $n$.

The heat equation that is often presented in physics as the prototype of the diffusion equation is written, as follows [30]:

$$
\partial u(x, t) / \partial t=\mathcal{D} \partial^{2} u(x, t) / \partial x^{2},
$$

where the diffusion coefficient is $\mathcal{D}=\lambda / \rho C$, with $\lambda, \rho$ and $C$ being, respectively, the thermal conductivity, the density, and the specific heat capacity of the material.

\subsubsection{Telegraph Equation}

A method that highlights the links between the wave equation and diffusion equation is to consider the telegraph equation. The telegraph equation is employed in various fields of physics, such as electricity, elasticity, and viscoelasticity, etc. Historically, it was established by Heaviside [31] for the transmission of electrical signals over lines (telegraph wires). The line is modeled by a two-port network characterized by the following constant quantities which are given per unit length: resistance $R$, inductance $L$, capacity $C$, and leakance $G$. The coupled relations between voltage $V(x, t)$ and current $I(x, t)$ are the 
Kirchhoff circuit laws that deal with the conservation of current and the conservation of energy:

$$
\partial V(x, t) / \partial x=-L \partial I(x, t) / \partial t-R I(x, t), \quad \text { and } \quad \partial I(x, t) / \partial x=-C \partial V(x, t) / \partial t-G V(x, t),
$$

leading to the telegraph equation :

$$
\partial^{2} f(x, t) / \partial x^{2}-L C \partial^{2} f(x, t) / \partial t^{2}=(R C+G L) \partial f(x, t) / \partial t+R G f(x, t),
$$

where $f(x, t)$ is $V(x, t)$ or $I(x, t)$. On the left-hand side of this equation, we recognize the terms of the propagation equation, while the right-hand terms govern distortion and attenuation of the wave. Therefore, it is a wave equation that takes into account the parameters of the medium that determine the propagation speed and the transport parameters. Note that we find the heat equation when $L=0$ and $G=0$, and when we identify $V$ with the temperature and $I$ with the heat flow.

The telegraph equation can also be considered to be a simple generalization of the diffusion equation. It was introduced to overcome the conceptual drawbacks that are related to the infinite speed inherent to the diffusion equation. Thus, it derives from the diffusion equation when the Fick's law is replaced by one of its generalizations, namely Maxwell-Cattaneo's law, which takes the rate of the flow into account [32]:

$$
\tau \partial \mathbf{q} / \partial t+\mathbf{q}=-k \nabla n
$$

Thus, we obtain

$$
\partial^{2} n / \partial t^{2}+\tau^{-1} \partial n / \partial t=k \tau^{-1} \nabla^{2} n
$$

Finally, another way of looking at the telegraph equation is to consider it as a nondissipative wave equation in which viscous and thermal damping have been added by hand:

$$
\partial^{2} n / \partial t^{2}+a \partial n / \partial t-c^{2} \partial^{2} n / \partial x^{2}=0,
$$

where $c$ is the speed of wave and $a$ is the attenuation coefficient.

As pointed out in [33], even though these last two Equations (35) and (36) have the same mathematical form, they are different in nature. Indeed, (35) is built according to the prescriptions that are given above, whereas (36) is not. In many applications, the introduction of wave damping is done by hand, i.e., without a proven scientific justification. For example, here the coefficient $a$ that contains all our misunderstanding about the mechanisms of energy transfer between the wave is the propagative medium. The only justification for the term $a \partial n / \partial t$ is that the dispersion equation leads to complexvalued wave numbers that are associated with damped waves. We will see later that, in this case, the DM solves the conservation equation and Euler's equation that support (36).

\section{Application of DM to Diffusion Equation and Wave Equation}

In this section, we apply the Dirac method to the time-dependent diffusion and wave equations in fluid mechanics.

\subsection{Diffusion Equation}

Let the diffusion equation be:

$$
\frac{\partial y(x, t)}{\partial t}-\mathcal{D} \frac{\partial^{2} y(x, t)}{\partial x^{2}}=0
$$

where $\mathcal{D}$ is the diffusion coefficient.

Using relations (11) and (14), the square root of the differential operator $\partial_{t}-\mathcal{D} \partial_{x x}^{2}$ leads to a matrix fractional differential equation

$$
\left(\sigma_{j} \partial_{t}^{1 / 2}+i \mathcal{D}^{1 / 2} \sigma_{k} \partial_{x}\right) Y=0 \quad j \neq k,
$$


where $Y$ is a two component vector function, depending on variables $x$ and $t$.

Several solutions are possible following the choice of couples $(j, k)$. For this equation, the only relevant choices are those that lead to PDEs whose coefficients are real numbers, i.e., those for that $(j, k) \neq(1,3)$ and $(j, k) \neq(3,1)$.

3.1.1. $j=1$ and $k=2$

With the choice $j=1$ and $k=2$, (38) becomes the matrix equation

$$
\left[\left(\begin{array}{ll}
0 & 1 \\
1 & 0
\end{array}\right) \partial_{t}^{1 / 2}+i\left(\begin{array}{cc}
0 & -i \\
i & 0
\end{array}\right) \mathcal{D}^{1 / 2} \partial_{x}\right]\left(\begin{array}{l}
y_{1}(x, t) \\
y_{2}(x, t)
\end{array}\right)=0,
$$

or the system of the following two scalar equations

$$
\begin{aligned}
& \mathcal{D}^{-1 / 2} \frac{\partial^{1 / 2} y_{2}}{\partial t^{1 / 2}}+\frac{\partial y_{2}}{\partial x}=0 \\
& \mathcal{D}^{-1 / 2} \frac{\partial^{1 / 2} y_{1}}{\partial t^{1 / 2}}-\frac{\partial y_{1}}{\partial x}=0 .
\end{aligned}
$$

They generalize the transport equations that are highlighted by the factorization of the wave equation (51), in which the first-order derivative of time is replaced by a fractional derivative of order $1 / 2$. Note that (40) has been highlighted by Kulish and Lage by another method [34] in the particular case of the momentum equation, assuming constant and uniform viscosity and neglecting the convective inertia advection effects. In addition, Raghavan has also reported this equation without explaining the passage from the diffusion operator to the fractional equation involving its square root [35]. As the diffraction equation is not invariant by time reversal, (41) has no obvious interpretation.

From Fick's law, we show that the flow of $y_{1}$

$$
q(x, t)=-k \frac{\partial y_{1}(x, t)}{\partial x}
$$

at the boundary $x=0$ is such that:

$$
q(t)=-k \frac{\partial^{1 / 2} y_{1}(0, t)}{\partial t^{1 / 2}}
$$

thus leading to the boundary condition:

$$
y_{1}(0, t)=\frac{\partial^{-1 / 2} q(t)}{\partial t^{-1 / 2}} .
$$

This relation shows the memory effect that develops at $x=0$, i.e., the boundary condition $y_{1}(x=0, t)$ that is given by the integral of order $1 / 2$ of its flow, which takes the whole history of its gradient into account.

3.1.2. $j=3$ and $k=2$

This case leads to

$$
\left[\left(\begin{array}{cc}
1 & 0 \\
0 & -1
\end{array}\right) \partial_{t}^{1 / 2}+i\left(\begin{array}{cc}
0 & -i \\
i & 0
\end{array}\right) \mathcal{D}^{1 / 2} \partial_{x}\right]\left(\begin{array}{l}
y_{1}(x, t) \\
y_{2}(x, t)
\end{array}\right)=0
$$

or else

$$
\begin{aligned}
& \mathcal{D}^{-1 / 2} \frac{\partial^{1 / 2} y_{1}}{\partial t^{1 / 2}}+\frac{\partial y_{2}}{\partial x}=0 \\
& \mathcal{D}^{-1 / 2} \frac{\partial^{1 / 2} y_{2}}{\partial t^{1 / 2}}-\frac{\partial y_{1}}{\partial x}=0 .
\end{aligned}
$$


As we have seen in Section 2.3.2, the phenomenon of diffusion is only related to one physical entity. Therefore, these equations that link the quantities $y_{1}$ and $y_{2}$ have a priori no physical meaning within the framework of diffusion.

\subsection{Wave Equation in a Non-Dissipative Medium}

For an ideal fluid, the transport coefficients i.e., the viscosity coefficients and thermal conductivity are all zero. Therefore, propagation only depends on the density and compressibility modulus of the fluid. From these two parameters, we can define two physical quantities that are more significant for the wave propagation, namely the specific impedance $Z=\sqrt{\rho / K}$ and the wave velocity $c=\sqrt{1 / K \rho}$.

Let the equation of waves in one-dimensional (1D) space be :

$$
\left(\partial_{x x}^{2}-c^{-2} \partial_{t t}^{2}\right) \phi=0
$$

Dirac's method leads to the matrix equation

$$
\left(\sigma_{j} \partial_{x}+i c^{-1} \sigma_{k} \partial_{t}\right) V=0,
$$

where $V$ is a two-component vector function $V=\left(\phi_{1}(x, t), \phi_{2}(x, t)\right)^{t}$ (here the superscript ${ }^{t}$ means transpose).

3.2.1. $j=1, k=2$

In this case, (49) is written, as follows:

$$
\left(\begin{array}{cc}
0 & \partial_{x}+c^{-1} \partial_{t} \\
\partial_{x}-c^{-1} \partial_{t} & 0
\end{array}\right)\left(\begin{array}{l}
\phi_{1}(x, t) \\
\phi_{2}(x, t)
\end{array}\right)=0
$$

corresponding to the transport equations:

$$
\left(\partial_{x}-c^{-1} \partial_{t}\right) \phi_{1}(x, t)=0 \text { and }\left(\partial_{x}+c^{-1} \partial_{t}\right) \phi_{2}(x, t)=0 .
$$

In this context, it is interesting to note that a transport equation can be seen as a conservation expression of the quantity $\phi_{i}$ that is associated with its flow $c \phi_{i}$. Indeed, by posing $v=(c, 1)^{t}$, the equation

$$
\left(\partial_{x}+c^{-1} \partial_{t}\right) f(x, t)=0,
$$

can be written

$$
\left(\frac{d}{d v}\right) f(x, t)=0
$$

which shows that $f(x, t)$ is constant in the $v$ direction. Thus, in the case of propagation in a non-dissipative medium, the wave form that is conserved.

The solutions to (51)

$$
\phi_{1}=f_{-}(x+c t) \quad \text { and } \quad \phi_{2}=f_{+}(x-c t)
$$

are the well known terms of the d'Alembert's solution that results from the factorization of the wave equation, as follows:

$$
\left(\partial_{x x}^{2}-c^{-2} \partial_{t t}^{2}\right) \phi=\left(\partial_{x}-c^{-1} \partial_{t}\right)\left(\partial_{x}+c^{-1} \partial_{t}\right) \phi=0
$$

They lead to (51) if the following condition is met:

$$
\partial^{2} \phi / \partial x \partial t=\partial^{2} \phi / \partial t \partial x
$$


This shows that Dirac's method is more general than d'Alembert's method, since it does not require any additional condition on cross derivatives of the function $\phi$ to establish the Equations (51). This result states that the equations that are produced by DM are the factors $P(D)$ and $Q(D)$ introduced in (2), whose solutions are two modes of propagation that travel in opposite directions along the $O x$ axis. This also justifies that Dirac's method should be seen as a factorization method.

3.2.2. $j=3$ and $k=2$

With the couple $(j=3, k=2)$, we obtain the following equations:

$$
\begin{aligned}
& \partial_{x} \phi_{1}+c^{-1} \partial_{t} \phi_{2}=0 \\
& \partial_{x} \phi_{2}+c^{-1} \partial_{t} \phi_{1}=0,
\end{aligned}
$$

where the quantities $\phi_{1}$ and $\phi_{2}$ have the same physical dimension.

As an application of this result, the DM is applied to a wave in an ideal fluid. In this case, the propagation is described through variations in the acoustic velocity and pressure of the fluid linked by an impedance relationship. Here, the term impedance means that there is a relation of proportionality between $p$ and $v$, with the ratio $p / v$ being a complex number depending on the parameters of the fluid. For a plane wave, this relationship is

$$
\frac{p}{v}=\rho c \quad \text { with } \quad K \rho c^{2}=1 .
$$

By writing (57) in the form

$$
\frac{\partial \phi_{1}}{\partial x}+\frac{K \rho c^{2}}{c} \frac{\partial \phi_{2}}{\partial t}=0,
$$

one gets

$$
\frac{\partial \phi_{1}}{\partial x}+K \rho c \frac{\partial \phi_{2}}{\partial t}=0 .
$$

Therefore, when considering that $\phi_{1}$ and $\phi_{2}$ have the dimension of a velocity that we identify as the acoustic velocity and using relationships (59), we have:

$$
K \partial p / \partial t=-\partial v / \partial x .
$$

In the same way, (56) becomes

$$
\rho \partial v / \partial t=-\partial p / \partial x .
$$

The two Equations (62) and (63) are the constitutive equation and the Euler Equation (27) used to establish the wave equation.

Therefore, applied to the wave equation, the DM leads, on the one hand, to d'Alembert's solution and, on the other hand, to the system of the basic equations that are the law of conservation and the constitutive relation involved in the wave equation. In a way, Dirac's method represents the inverse operation of establishing the wave equation from the conservation equation and the constitutive relationship, as seen in Section 2.3.1.

3.2.3. Case $j=2$ and $k=3$

In this case, Equation (48) becomes

$$
\left(\left(\begin{array}{cc}
0 & -i \\
i & 0
\end{array}\right) \frac{\partial}{\partial x}+\frac{i}{c}\left(\begin{array}{cc}
1 & 0 \\
0 & -1
\end{array}\right) \frac{\partial}{\partial t}\right)\left(\begin{array}{l}
\phi_{1} \\
\phi_{2}
\end{array}\right)=0 .
$$


leading to the system of equations:

$$
\begin{aligned}
& \partial_{x} \phi_{2}-c^{-1} \partial_{t} \phi_{1}=0, \\
& \partial_{x} \phi_{1}-c^{-1} \partial_{t} \phi_{2}=0,
\end{aligned}
$$

These equations are analogous to (57) and (58), in which $c$ is replaced by $-c$ and they derive from the time-reversal invariance of the wave equation.

\subsection{Wave Equation in a Dissipative Medium}

When a wave propagates in a dissipative medium, it gives up part of its energy to this medium. The process responsible for this attenuation essentially depends on the interaction of the wave with the structure that represents the support in question. For an acoustic wave in a real fluid, attenuation has two causes: (i) the viscosity of the fluid and (ii) its thermal conductivity. The effects of viscosity essentially develop in areas of the fluid where the velocity gradients are important. It is, in particular, the case near the walls of a pipe in which the fluid flows or downstream of obstacles that hinder its movement and generate vortices. The effects of thermal conduction are due to heat exchanges between the fluid parts due to compression and expansion imposed on the fluid by the passage of the wave. These effects are negligible in liquids, but they must be taken into account in gases.

Viscous and thermal effects play a particularly important role in the propagation of acoustic waves in saturated porous media. In these media, because of the large specific surface area of the fluid-solid structure and the viscosity of the fluid that imposes a zero fluid-solid relative velocity at the interface, it is legitimate to consider that the velocity gradients are significant at any point of the medium volume. Consequently, the viscous effects develop throughout the volume of the material. In plastic foams or fibrous media saturated with gas, the density of the structure is high when compared with that of the gas. Therefore, it can be considered that, when an acoustic wave passes through a porous medium, only the gas is set in motion, while the solid structure remains at rest. Moreover, as the thermal conductivity of the fluid is small when compared with that of the solid structure, the latter acts as a thermostat, favoring the thermal exchanges between fluid and structure. In the equivalent fluid model [36-38], which is a simplified version of Biot's model $[39,40]$, these effects are taken into account in the density and compressibility of the fluid through two factors: the dynamic tortuosity and the dynamic compressibility. These two factors, which are the responses of the porous medium to the excitation due to the passage of the wave renormalize the density and the compressibility of the fluid in the sense that the interactions that develop in the fluid change the values of these parameters.

In a dissipative medium, the propagation of a wave is governed by the telegraph equation

$$
\left(\partial_{x x}^{2}-c^{-2} \partial_{t t}^{2}+a \partial_{t}\right) \phi=0,
$$

where $a$ is the damping coefficient. Applying DM leads to the matrix equation

$$
\left(\sigma_{j} \partial_{x}+i c^{-1} \sigma_{k} \partial_{t}+a^{1 / 2} \sigma_{l} \partial_{t}^{1 / 2}\right) V=0 \quad \text { with } \quad j \neq k \neq l
$$

where $V=\left(\phi_{1}, \phi_{2}\right)^{t}$.

3.3.1. Case $j=3, k=2, l=1$

For the triplet $(j, k, l)=(3,2,1)$, the following system is obtained:

$$
\begin{aligned}
\partial_{x} \phi_{1}+c^{-1} \partial_{t} \phi_{2}+a^{1 / 2} \partial_{t}^{1 / 2} \phi_{2} & =0 \\
-\partial_{x} \phi_{2}+c^{-1} \partial_{t} \phi_{1}+a^{1 / 2} \partial_{t}^{1 / 2} \phi_{1} & =0 .
\end{aligned}
$$

In these equations, the third term represents a fractional derivative of the order $\alpha=1 / 2$. Here, again, we can see the advantage that is offered by the DM, which allows for 
the formalism of fractional calculation to be introduced naturally. Fractional derivatives of the order of $1 / 2$ take the effects of viscosity and thermal conductivity of the fluid into account. Thus, they model the memory effects of the processes involved through Equations (69) and (70).

If we pose $\phi_{1}=v(x, t)$, then $\phi_{2}=p(x, t) / Z$, where $Z$ is the impedance, (69) becomes

$$
\frac{\partial v}{\partial x}+\frac{1}{c Z} \frac{\partial p}{\partial t}+\frac{a^{1 / 2}}{Z} \frac{\partial^{1 / 2} p}{\partial t^{1 / 2}}=0 .
$$

For a plane wave in a fluid, then $Z=\rho c$ and (71) becomes:

$$
\frac{\partial v}{\partial x}+\frac{1}{\rho c^{2}} \frac{\partial p}{\partial t}+\frac{a^{1 / 2} c}{\rho c^{2}} \frac{\partial^{1 / 2} p}{\partial t^{1 / 2}}=0 .
$$

Taking into account the relation $K \rho c_{0}^{2}=1$ and the property (18) of the fractional derivative, (72) is finally written, as follows:

$$
\frac{\partial v}{\partial x}+K\left(\delta(t)+a^{1 / 2} c \Phi_{1 / 2}(t)\right) * \frac{\partial p}{\partial t}=0 .
$$

where $*$ denotes the convolution product $f(t) * g(t)=\int f(t-\tau) g(\tau) d \tau$ and $\delta(t)$ is the Dirac's distribution. Thus, the term $a \partial \phi / \partial t$ that is introduced in the wave equation modifies the coefficient of compressibility of the fluid that can now be noted

$$
K(t)=K\left(\delta(t)+a^{1 / 2} c \frac{\partial^{-1 / 2}}{\partial t^{-1 / 2}}\right) .
$$

With the same notations, (70) becomes:

$$
-\frac{\partial p}{\partial x}+\frac{Z}{c} \frac{\partial v}{\partial t}+a^{1 / 2} Z \frac{\partial^{1 / 2} v}{\partial t^{1 / 2}}=0 .
$$

For a plane wave, we find

$$
\begin{aligned}
-\frac{\partial p}{\partial x} & =\rho \frac{\partial v}{\partial t}+a^{1 / 2} \rho c \frac{\partial^{1 / 2} v}{\partial t^{1 / 2}} \\
& =\rho\left(\delta(t) *-a^{1 / 2} c \frac{\partial^{-1 / 2}}{\partial t^{-1 / 2}}\right) \frac{\partial v}{\partial t}
\end{aligned}
$$

These results (74) and (77) show that, in the DM framework, the telegraph equation leads to modified Euler and mass conservation equations. The wave/fluid interactions that re modeled by the term $a \partial \phi / \partial t$ are taken into account in the density and compressibility of the fluid. These two parameters can now be seen as time operators that act, respectively, on $\partial v / \partial t$ and $\partial p / \partial t$. They are the sum of two contributions: (i) an instantaneous action $\delta(t)$ and (ii) a memory action represented by a quantity proportional to the fractional derivative $\partial^{-1 / 2} / \partial t^{-1 / 2}$. In the frequency domain, they act as susceptibilities that quantify the response of the fluid to wave solicitations. In a real fluid, $a$ is the sum of the contributions of viscous and thermal attenuation. Zwikker and Kosten have shown [41] that these contributions can be decoupled: the viscous effects act on the density of the gas while the thermal ones act on its compressibility.

In integral form, relationship (77) becomes

$$
\rho \frac{\partial v(x, t)}{\partial t}=-\frac{\partial p(x, t)}{\partial x}+\frac{a^{1 / 2} c \rho}{\Gamma(\pi / 2)} \int \frac{\partial v(x, \tau) / \partial \tau}{\sqrt{t-\tau}} d \tau
$$

This is Euler's equation of a unit volume of fluid that is subjected to the pressure exerted by the acoustic wave and the force of viscosity that develops between parts of the fluid moving at different speeds. This force is expressed by a fractional derivative of the 
order of $1 / 2$, which models the memory effect. It is due to the delayed development of the boundary layer with the changing field of acceleration in a fluid. It is analogous, for a fluid, to the force that was highlighted by Basset [42] and Boussinesq [43] in the case of a sphere in a non-stationary motion within a viscous fluid given e.g., in [44,45].

3.3.2. Case $j=1, k=2, l=3$

With the triplet $(j, k, l)=(1,2,3)$, we obtain the following matrix equation :

$$
\left[\left(\begin{array}{ll}
0 & 1 \\
1 & 0
\end{array}\right) \partial_{x}+\frac{i}{c}\left(\begin{array}{cc}
0 & -i \\
i & 0
\end{array}\right) \partial_{t}+a^{1 / 2}\left(\begin{array}{cc}
1 & 0 \\
0 & -1
\end{array}\right) \partial_{t}^{1 / 2}\right]\left(\begin{array}{c}
\phi_{-} \\
\phi_{+}
\end{array}\right)=0
$$

corresponding to the system of differential equations:

$$
\left\{\begin{array}{l}
a^{1 / 2} \partial_{t}^{1 / 2} \phi_{-}+\left(\partial_{x}+\frac{1}{c} \partial_{t}\right) \phi_{+}=0 \\
\left(\partial_{x}-\frac{1}{c} \partial_{t}\right) \phi_{-}-a^{1 / 2} \partial_{t}^{1 / 2} \phi_{+}=0 .
\end{array}\right.
$$

One immediately checks that the functions $\phi_{+}$and $\phi_{-}$are solutions to Equation (67) corresponding to modes that propagate along the $O x$ axis in opposite directions. By comparing Equations (50) and (80), we see that the term $a \partial_{t}^{1 / 2} \phi_{ \pm}$modifies the transport equations of the wave in a non-dissipative medium and it represents the coupling between the two modes $\phi_{+}$and $\phi_{-}$. The sum and difference of the Equations (80) lead to the following relationships

$$
\begin{aligned}
c \partial_{x} \varrho+c^{-1} \partial_{t} j & =+a^{1 / 2} \partial_{t}^{1 / 2} j \\
c \partial_{t} \varrho+\partial_{x} j & =-a^{1 / 2} c \partial_{t}^{1 / 2} \varrho
\end{aligned}
$$

where $\varrho=\phi_{+}+\phi_{-}$and $j=c\left(\phi_{+}-\phi_{-}\right)$.

Equation (82) is the one-dimensional analogue of the equation of continuity. It expresses that, in a real fluid, the amplitudes $\phi_{+}$and $\phi_{-}$are not conserved and the wave damping results from memory effects due to the viscosity and thermal conductivity developing in the fluid. In this respect, it should be remembered that, in general, memory effects have a strong influence on transport phenomenon. As for (81), it is a constitutive equation. This shows that Dirac's method makes it possible to deduce from the telegraph equation information not accessible by conventional approaches.

\section{Discussion and Conclusions}

In this paper, we have applied Dirac's method to some classical equations of fluid mechanics, namely, the wave equation, the scattering equation, and the telegrapher's equation. We have shown that this method has several advantages. In the wave equation, it leads to the well-known d'Alembert's solutions. However, it is more general than $d^{\prime}$ Alembert's factorization, since it does not impose a condition on the cross-derivatives with respect to time and space. A new result is that it "decomposes" the wave equation, which restores the starting equations according to the following scheme:

$$
\left(\begin{array}{c}
\text { balance equation } \\
\text { constitutive relationship }
\end{array}\right) \stackrel{\text { any cl. method }}{\stackrel{\text { Dirac method }}{\leftrightarrows}}\left(\begin{array}{c}
\text { wave } \\
\text { equation }
\end{array}\right)
$$

Thus, Dirac's method is the opposite of any classical method, as described in Section 2.3.1. Its interest comes from the fact that there are few mathematical tools that are capable of giving such a result. Therefore, it is complementary to the Lagrangian method. Indeed, it is known that the Lagrangian method is widely applied to obtain equations of motion for systems in which interactions are entangled or for which physics principles other than symmetries are difficult to implement. Dirac's method, which is applied to 
the Euler equations deduced from the Lagrangian, makes it possible to extract the laws governing the phenomenon of propagation.

With respect to the diffusion equation, Dirac's method has established a fractional differential equation that generalizes the transport equation with a fractional term, without the addition of further assumptions. In this equation, the fractional derivative governs the memory effects that develop during the diffusion process.

The results for the application of DM to the telegrapher's equation are both new and promising for applications. Indeed, on the one hand, a generalization of the Euler equation and the constitutive relation in the form of fractional PDEs has been obtained. These equations are new in the sense that they are difficult to establish from the principles of physics. Two succeptibilities of the fluid have been defined thanks to the fractional calculation: its dynamic density and its dynamic compressibility. In this formalism, these two parameters are interpreted as time-dependent operators that act on the wave that excites the fluid. Each of them is the sum of two contributions: an instantaneous action and a memory term. The most striking feature of Euler's equation is the highlighting of a term that is similar to the one found by Boussinesq and Basset in the case of a solid sphere in a fluid in non-stationary motion. On the other hand, transport equations have been established, which, considered as conservation equations, show that the amplitudes of the propagative modes are not conserved and they constitute a wave splitting solution for the problem of a wave in a viscous fluid.

Dirac's method, as illustrated in the above-mentioned examples, offers interesting perspectives for the physical interpretation of mathematical results, especially in the framework of fractional calculus.

Author Contributions: Writing-Original Draft Preparation, Z.E.A.F., M.F., E.O. and C.D.; Analysis, analytical calculations and processing of results, Z.E.A F., M.F., E.O. and C.D.; Writing-Review \& Editing, Z.E.A.F., M.F., E.O. and C.D.; Resources,Z.E.A.F, M.F., E.O. and C.D. All authors have read and agreed to the published version of the manuscript.

Funding: This research received no external funding.

Institutional Review Board Statement: Not applicable.

Informed Consent Statement: Not applicable.

Data Availability Statement: Not applicable.

Conflicts of Interest: The authors declare no conflict of interest.

\section{References}

1. Matsuura, S. Factorization of Differential Operators and decomposition of solution of Homogeneous Equations. Osaka Math. J. $1963,15,213-231$.

2. Schrödinger, E. A method of determining quantum-mechanical eigenvalues and eigenfunctions. Proc. R. Irish Acad. 1940, 46, 9-16.

3. Schrödinger, E. Further studies on solving eigenvalue problems by factorization. Proc. R. Irish Acad. 1940, 46, 183-206.

4. Dirac, P.A.M. Principles of Quantum Mechanics, 2nd ed.; Clarendon Press: Oxford, UK, 1935.

5. Infeld, L. A new treatment of some eigenvalue problems. Phys. Rev. 1941, 59, 737-747. [CrossRef]

6. Infeld, L. A generalization of the factorization method of solving eigenvalue problems. Trans. R. Soc. Can. 1942, 36, 7-18.

7. Infeld, L.; Schild, A. A note on the Kepler problemin a space of constant negative curvature. Phys. Rev. 1945, 67, 121-122. [CrossRef]

8. Infeld, L. Recurrence formulas for coulomb wave function. Phys. Rev. 1947, 72, 1125. [CrossRef]

9. Hull, T.E.; Infeld, L. The factorization method, hydrogen intensities and related problems. Phys. Rev. 1948, 74, 905-909. [CrossRef]

10. Infeld, L. The factorization method and its application to differential equations in theoretical physics. Proc. Symp. Appl. Math. Am. Math. Soc. 1950, II, 58-65.

11. Infeld, L.; Hull, T.E. The factorization method. Rev. Mod. Phys. 1951, 23, 21-68. [CrossRef]

12. Mielnik, B. Factorization method and new potentials with the oscillator spectrum. J. Math. Phys. 1984, 25, 3387-3389. [CrossRef]

13. Mielnik, B.; Rosas-Ortiz, O. Factorization: Little or great algorithm? J. Phys. Math. Gen. 2004, 37, 10007-10035. [CrossRef]

14. Gendenshtein, L.E. Derivation of exact spectra of the Schrödinger equation by means of SUSY. JETP Lett. 1983, 38, 356-359.

15. Smirnov, Y.F. On factorization and algebrization of difference equations of hypergeometric type. In Proceedings of the International Workshop on Orthogonal Polynomials in Matematical Physics, Leganes, Spain, 24-26 June 1996. 
16. Smirnov, Y.F. Factorization method: New aspects. Rev. Mex. Fis. 1999, 45, 1-6.

17. Rosu, H.C. One-parameter families of supersymmetric isospectral potentials from Riccati solutions in function composition form. Ann. Phys. 2014, 343, 87-102. [CrossRef]

18. Rosu, H.C.; Mancas, S.C.; Chen, P. The classical harmonic oscillator with Dirac-like parameters and possible applications. J. Phys. A Math. Gen. 2004, 37, 11699-11710. [CrossRef]

19. Dirac, P.A.M. The quantum theory of the electron. Proc. R. Soc. Lond. A 1928, 117, 610-624.

20. Babusci, D.; Dattoli, G.; Quattromini, M. Relativistic equations with fractional and pseudodifferential operators. Phys. Rev. A 2011, 83, 062109. [CrossRef]

21. Babusci, D.; Dattoli, G.; Quattromini, M. A note on the extension of the Dirac method. Appl. Math. Comput. 2011, $218,1495-1497$. [CrossRef]

22. Babusci, D.; Dattoli, G. Dirac factorization method and the harmonic oscillator. arXiv 2012, arXiv:1203.2526.

23. Babusci, D.; Dattoli, G. Dirac factorization and fractional calculus. arXiv 2012, arXiv:1209.2276.

24. Dattoli, G.; Torre, A. Root operators and "evolution" equations. Mathematics 2015, 3, 690-726. [CrossRef]

25. Torre, A.; Dattoli, G.; Quattromini, M.; Babusci, P.E.R.D. Extension of the Dirac Factorization and relevant Applications. Bull. TICMI 2014, 18, 52-66.

26. Depollier, C.; Allard, J.F.; Lauriks, W. Biot Theory and stress-strain equations in porous sound-absorbing. J. Acoust. Soc. Am. 1988 83, 2277-2279. [CrossRef]

27. Bogoliubov, N.N.; Shirkov, D.V. Introduction to the Theory of Quantized Fields. In Interscience Monographs in Physics and Astronomy; John Wiley \& Sons: Hoboken, NJ, USA, 1959.

28. Johnson, D.L.; Plona, T.J. Recents developpments in the acoustic properties of porous media. In Enrico Fermi School: Frontiers of Physical Acoustics; Sette, T., Ed.; Cours XCII: Amsterdam, The Netherlands, 1986.

29. Temkin, S. Elements of Acoustics; John Wiley \& Sons: New York, NY, USA, 1981.

30. Kress, R. Linear Integral Equations; Springer: New York, NY, USA, 1999.

31. Heaviside, O. Electromagnetic Theory; Ernest Benn Limited: London, UK, 1893.

32. Cattaneo, C. Sulla conduzione del calore. Atti Semin. Mat. E Fis. Modena 1948, 3, 83-101.

33. Zhang, D.; Ostoja-Strarzewski, M. Telegraph equation: Two types of harmonic waves, a discontinuity wave, and a spectral finite element. Acta Mech. 2019, 230, 1725-1743. [CrossRef]

34. Kulish, V.V.; Lage, J.L. Application of Fractional Calculus to Fluid Mechanics. J. Fluids Eng. 2002, 124, 803-806. [CrossRef]

35. Raghavan, R. Fractional diffusion: Performance of fractured wells. J. Pet. Sci. Eng. 2012, 92, 167-173. [CrossRef]

36. Johnson, D.L.; Koplik, J.; Dashen, R. Theory of dynamic permeability and tortuosity in fluid-saturated porous media. J. Fluid Mech. 1987, 176, 379-402. [CrossRef]

37. Champoux, Y.; Allard, J.-F. Dynamic tortuosity and bulk modulus in air-saturated porous media. J. Appl. Phys. 1991, 70, 1975-1979. [CrossRef]

38. Lafarge, D.; Lemarinier, P.; Allard, J.-F.; Tarnow, V. Dynamic compressibility of air in porous structures at audible frequencies. J. Acoust. Soc. Am. 1997, 102, 1995-2006. [CrossRef]

39. Biot, M.A. Theory of propagation of elastic waves in a fluid-saturated porous solid. I. J. Acoust. Soc. Am. 1956, 28, 168-178. [CrossRef]

40. Biot, M.A. Theory of propagation of elastic waves in a fluid-saturated porous solid. II. J. Acoust. Soc. Am. 1956, 28, 179-191. [CrossRef]

41. Zwikker, C.; Kosten, C.W. Sound Absorbing Materials; Elsevier: Amsterdam, The Netherlands, 1949.

42. Basset, A.B. Treatise on Hydrodynamics; Deighton, Bell and Co.: Cambridge, UK, 1888.

43. Boussinesq, J.V. Sur la résistance qu'oppose un fluide indéfini au repos, sans pesanteur, au mouvement varié d'une sphère solide qu'il mouille sur toute sa surface, quand les vitesses restent bien continues et assez faibles pour que leurs carrés et produits soient négligeables. Comptes Rendus L'AcadÉmie Des Sci. 1885, 100, 935-937.

44. Michaelides, E.E. Hydrodynamic Force and Heat/Mass Transfer $>$ From Particles, Bubbles, and Drop-The Freeman Scholar Lecture. J. Fluids Eng. 2003, 125, 209-238. [CrossRef]

45. Buonocore, S.; Sen, M.; Semperlotti, F. A fractional-order approach for transient creeping flow of spheres. AIP Adv. $2019,9,085323$. [CrossRef] 